Fig. 1 Altered social behaviour-induced changes in the amygdala. Under baseline activity the amygdala of germ-free mice is an activated state. Transcriptomic analysis demonstrated an upregulation of several immediate early response genes such as Fos, Fosb, Egr2 or $\mathrm{Nr} 4 \mathrm{a} 1$ in association with increased CREB signalling in GF mice (see [5] for full details). Moreover, when a germ-free mouse is introduced to a social stimulus the normal transcriptional pathway recruitment is absent but instead genes involved in alternative splicing are enriched (see [6] for full details of genes affected)

However, GF mice displayed a strikingly different pattern of amygdala gene activity in response to social interaction [6] (Fig. 1). In particular, the dynamic, stimulus-dependent transcriptional regulation seen in controls was attenuated and replaced by a marked increase in expression of splicing factors and alternative exon usage. This reveals a potential molecular basis for how the host microbiome is crucial for a normal behavioural response during social interaction. Moreover, social behaviour was correlated with the amygdala gene-expression response. These results reveal one of the key steps leading from absence of bacteria during brain development to a phenotype associated with reduced sociability in adulthood in mice. These data thus enhance our understanding of the link between the microbiome and brain health and neurodevelopmental disorders such as autism spectrum disorders. Future studies will be needed to determine what are the exact microbial signals that regulate alternative splicing events in the amygdala and whether they can be harnessed for therapeutic benefit.

\section{FUNDING AND DISCLOSURE}

The authors are supported by Science Foundation Ireland (Grant Nos. SFI/12/RC/2273). They have had research support from
Mead Johnson, Cremo, 4D Pharma, Suntory Wellness, Dupont and Nutricia.

\section{ACKNOWLEDGEMENTS}

The authors would like to thank Dr. Roman Stilling for his stimulating insights into amygdala-microbiome interactions that form the basis of this short article and Dr. Stilling and Dr. Kiran Sandhu for assistance with Fig. 1.

\section{ADDITIONAL INFORMATION}

Publisher's note: Springer Nature remains neutral with regard to jurisdictional claims in published maps and institutional affiliations.

\section{REFERENCES}

1. Allen AP, Dinan TG, Clarke G, Cryan JF. A psychology of the human brain-gutmicrobiome axis. Soc Personal Psychol Compass. 2017;11:e12309.

2. Desbonnet L, Clarke G, Shanahan F, Dinan TG, Cryan JF. Microbiota is essential for social development in the mouse. Mol Psychiatry. 2014;19:146-8.

3. Cowan CSM, Hoban AE, Ventura-Silva AP, Dinan TG, Clarke G, Cryan JF. Gutsy moves: the amygdala as a critical node in microbiota to brain signaling. Bioessays. 2018:40:1700172.

4. Luczynski P, McVey Neufeld KA, Oriach CS, Clarke G, Dinan TG, Cryan JF. Growing up in a bubble: using germ-free animals to assess the influence of the gut microbiota on brain and behavior. Int J Neuropsychopharmacol. 2016;19:pii: pyw020.

5. Stilling RM, Ryan FJ, Hoban AE, Shanahan F, Clarke G, Claesson MJ, et al. Microbes \& neurodevelopment-absence of microbiota during early life increases activity-related transcriptional pathways in the amygdala. Brain Behav Immun. 2015;50:209-20.

6. Stilling RM, Moloney GM, Ryan FJ, Hoban AE, Bastiaanssen TF, Shanahan F, et al. Social interaction-induced activation of RNA splicing in the amygdala of microbiome-deficient mice. eLife. 2018;7:pii: e33070.

\title{
Novel models of drug relapse and craving after voluntary abstinence
}

\author{
Marco Venniro ${ }^{1}{ }^{1}$, Daniele Caprioli ${ }^{2,3}$ and Yavin Shaham ${ }^{1}$ \\ Neuropsychopharmacology (2019) 44:234-235; https://doi.org/10.1038/s41386-018-0196-4
}

Relapse to drug use during abstinence is a core feature of addiction. Since the 1980s, this clinical scenario has been studied using animal models where relapse is assessed after experimenterimposed cessation of drug self-administration by either extinction of the drug-reinforced responding or homecage forced abstinence [1]. However, despite strides toward understanding neuronal mechanisms of relapse in these models, treatment options remain largely unchanged. One potential reason for this state-of-affairs is that animal models of addiction rarely incorporate voluntary aspects of human abstinence, which often occurs due to the availability of alternative nondrug rewards (e.g., employment and supportive social environment). This is exemplified in contingency management where nondrug rewards (monetary vouchers), given in exchange for being drug-free, can maintain abstinence for many months. However, when contingency management discontinues, most drug users relapse.

Based on these considerations, we introduced a contingency management-based relapse model, where we achieve long-lasting voluntary abstinence prior to the relapse tests by giving rats mutually exclusive choices between a drug and palatable food [2]. In the initial study, we trained male rats to self-administer the food and methamphetamine in established addiction models-escalation and DSM-IV-based-and found that rats will voluntarily abstain from drug self-administration for at least 3 weeks and then show incubation of methamphetamine craving (time-dependent increases in drug seeking during abstinence) [2]. Subsequently, we

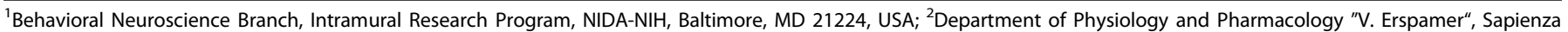
University of Rome, Rome, Italy and ${ }^{3}$ Santa Lucia Foundation (IRCCS Fondazione Santa Lucia), Rome, Italy

Correspondence: Marco Venniro (venniro.marco@nih.gov)

Published online: 11 September 2018 
Social-choice self-administration chamber

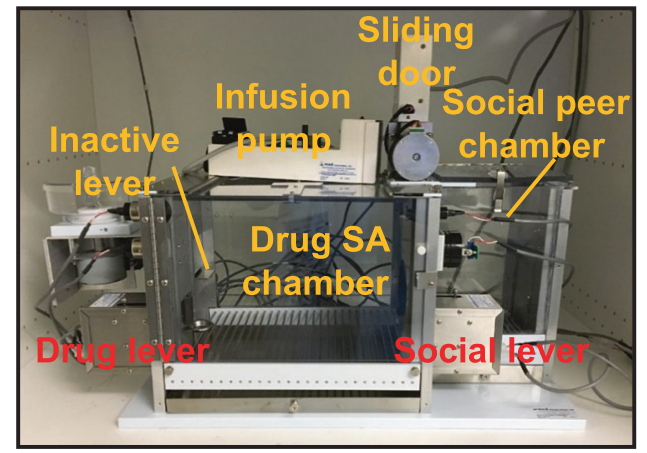

Fig. 1 Custom-made social-choice self-administration apparatus. For instructions on how to build the apparatus, see [6], and technical advice is available upon request from the authors

demonstrated the generality of food-choice voluntary abstinence to female rats and to heroin, and that surprisingly voluntary abstinence prevents incubation of heroin craving [3]. In mechanistic studies, we found a role of dorsomedial striatum neuronal ensembles in incubation of methamphetamine craving after voluntary abstinence [4], and a role of glutamatergic projections from the anterior insular cortex to central amygdala in relapse after voluntary abstinence [5].

However, the use of palatable food as the nondrug reward may limit the model's clinical translation. This is because for most humans, the rewards that compete with drugs are primarily social (family and employment). Based on this consideration, we recently introduced a newer voluntary abstinence model that involves choices between a drug and operant access to social interaction [6] (Fig. 1). We found that rats trained in established addiction models-escalation, DSM-IV-based, and intermittent access-will voluntarily abstain when given mutually exclusive choices between methamphetamine or heroin versus social interaction. This effect was independent of their 'addiction score', persisted through 4 weeks of forced abstinence, and could only be reversed by delay or punishment of the social reward. We also found that social-choice-induced voluntary abstinence prevents the emergence of incubation of methamphetamine craving, even 1 month after cessation of the social choice. This protective effect was associated with activation (assessed by the activity marker Fos) of inhibitory central amygdala PKC $\delta$-expressing neurons and decreased neuronal activity in the anterior insular cortex [6].

In conclusion, we introduced two novel models of choice-based voluntary abstinence and demonstrated the profound protective effects of positive social interaction on drug addiction and relapse in rat models. Our findings support wider implementation of social-based behavioral treatments, which include not only the established community reinforcement approach, but also socialbased psychotherapies and family-based social support systems to provide social support before and during drug-seeking episodes.

\section{ACKNOWLEDGEMENTS}

The research was supported by the Intramural Research Program of NIDA (YS), NARSAD Distinguished Investigator Grant Award (YS), and a fellowship from the NIH Center on Compulsive Behaviors (MV).Competing interests: The authors declare no competing interests

Publisher's note: Springer Nature remains neutral with regard to jurisdictional claims in published maps and institutional affiliations.

\section{REFERENCES}

1. Venniro M, Caprioli D, Shaham Y. Animal models of drug relapse and craving: from drug priming-induced reinstatement to incubation of craving after voluntary abstinence. Prog Brain Res. 2016;224:25-52.

2. Caprioli D, Venniro M, Zeric T, Li X, Adhikary S, Madangopal R, et al. Effect of the novel positive allosteric modulator of metabotropic glutamate receptor 2 AZD8529 on incubation of methamphetamine craving after prolonged voluntary abstinence in a rat model. Biol Psychiatry. 2015;78:463-73.

3. Venniro M, Zhang M, Shaham Y, Caprioli D. Incubation of methamphetamine but not heroin craving after voluntary abstinence in male and female rats. Neuropsychopharmacology. 2017;42:1126-35.

4. Caprioli D, Venniro M, Zhang M, Bossert JM, Warren BL, Hope BT, Shaham Y. Role of dorsomedial striatum neuronal ensembles in incubation of methamphetamine craving after voluntary abstinence. J Neurosci. 2017;37:1014-27.

5. Venniro M, Caprioli D, Zhang M, Whitaker LR, Zhang S, Warren BL, et al. The anterior insular cortex-->central amygdala glutamatergic pathway is critical to relapse after contingency management. Neuron. 2017;96:414-27 e418.

6. Venniro M, Zhang M, Caprioli D, Hoots JK, Golden SA, Heins C, et al. Volitional social interaction prevents drug addiction in rat models. Nature Neuroscience. 2018 (In press).

\section{An emerging epigenetic framework of systemic and central mechanisms underlying stress-related disorders}

Carla Nasca ${ }^{1}$, Natalie Rasgon ${ }^{1,2}$ and Bruce McEwen ${ }^{1}$

Neuropsychopharmacology (2019) 44:235-236; https://doi.org/10.1038/s41386-018-0227-1

Advances in translational neuroscience are pointing to a new paradigm for conceptualizing diagnosis and treatment of major morbidities, such as major depressive disorders (MDD), diabetes, and dementia [1]. Here we explore the emerging framework that focuses on the epigenetic actions of metabolic mediators on regulation of gene expression in brain regions controlling cognition and emotion as an approach to examine the systemic, as well as neural bases, of stress-related CNS disorders.

\footnotetext{
${ }^{1}$ Harold and Margaret Milliken Hatch Laboratory of Neuroendocrinology, The Rockefeller University, New York, USA and ${ }^{2}$ Center for Neuroscience in Women's Health, Stanford University, Palo Alto, CA 91304, USA

Correspondence: Carla Nasca (cnasca@rockefeller.edu) or Bruce McEwen (mcewen@rockefeller.edu)
}

Published online: 7 October 2018 